\section{Antioxidative Kapazität}

A. C. Sewell

Institut für Medizinische Diagnostik GmbH, Bioscientia, Ingelheim, Deutschland

Synonym(e) TEAC; Trolox-Äquivalent

Englischer Begriff trolox equivalent; antioxidative capacity

Definition Parameter der antioxidativen Kapazität.
Beschreibung Mithilfe des Trolox-Äquivalents kann die antioxidative Kapazität einer Probe unterschiedlicher Herkunft (z. B. Blutplasma, Lebensmittel) angegeben werden. Bei der Bestimmung dient das $>$ Vitamin E-Derivat Trolox als Referenz. Der TEAC-Wert wird häufig zum Vergleich bei polyphenolhaltigen Proben (z. B. Wein, Säfte) herangezogen.

\section{Literatur}

Miller NJ, Rice-Evans C et al (1993) A novel method for measuring antioxidant capacity and its application to monitoring the antioxidant status in premature infants. Clin Sci 84:407-412 